\title{
ON SPACES OF MAPS \\ BETWEEN COMPLEX PROJECTIVE SPACES
}

\author{
JESPER MICHAEL M $\phi L L E R$
}

\begin{abstract}
When $1 \leqslant m \leqslant n$, the space $M\left(P^{m}, P^{n}\right)$ of maps of complex projective $m$-space $P^{m}$ into complex projective $n$-space $P^{n}$ has a countably infinite number of components enumerated by degrees of maps in $H^{2}\left(P^{m} ; \mathbf{Z}\right)$. By calculating their $(2 n-2 m+1)$-dimensional integral homology group we show that two components of $M\left(P^{m}, P^{n}\right)$ are homotopy equivalent if and only if their associated degrees have the same absolute value.
\end{abstract}

1. Introduction and statement of result. Let $P^{n}$ denote the complex projective $n$-space. For $1 \leqslant m \leqslant n$, consider the space $M\left(P^{m}, P^{n}\right)$ of (continuous) maps of $P^{m}$ into $P^{n}$. equipped with the compact-open topology. $M\left(P^{m}, P^{n}\right)$ has a countably infinite number of (path-)components, for the homotopy classes of maps of $P^{m}$ into $P^{n}$ are classified by their degrees in $H^{2}\left(P^{m} ; \mathbf{Z}\right) \cong \mathbf{Z}$. For each integer $k \in \mathbf{Z}$, let $M_{k}\left(P^{m}, P^{n}\right)$ denote the component of $M\left(P^{m}, P^{n}\right)$ consisting of maps of degree $k$. The object of this paper is to prove the following

THEOREM. The $(2 n-2 m+1)$-dimensional integral homology group of $M_{k}\left(P^{m}, P^{n}\right)$, $1 \leqslant m \leqslant n$, is cyclic of infinite order for $k=0$ and cyclic of order $\left(\begin{array}{c}n+1 \\ m\end{array}\right)|k|^{m}$ for $k \neq 0$ :

$$
H_{2 n-2 m+1}\left(M_{k}\left(P^{m}, P^{n}\right)\right) \cong \mathbf{Z} /\left(\begin{array}{c}
n+1 \\
m
\end{array}\right)|k|^{m} \mathbf{Z} \text {. }
$$

Some special cases of this result already occur in the literature. See [4, 2], for the case $n=m=1$ and [5] for the case $n=m$.

An easily derived consequence of the Theorem is the following

Corollary. Two components $M_{k}\left(P^{m}, P^{n}\right)$ and $M_{l}\left(P^{m}, P^{n}\right)$ of $M\left(P^{m}, P^{n}\right)$ are homotopy equivalent if and only if $|k|=|l|$.

This corresponds to result obtained by V. L. Hansen $[1,2,3]$ for the case of spaces of maps of $n$-manifolds into the $n$-sphere.

ACKNOWLEDGEMENTS. I am very grateful to V. L. Hansen for posing the problem that led to this paper and to the referee whose constructive remarks improved the original argument considerably.

2. Restriction fibrations. For any two spaces $X$ and $Y$, we shall write $M(X, Y)$ for the space of maps of $X$ into $Y$ equipped with the compact-open topology, and for

Received by the editors February 21, 1983 and, in revised form, August 12, 1983.

1980 Mathematics Subject Classification. Primary 55P15, Secondary 58D15.

Key words and phrases. Homotopy classification of components of mapping spaces, restriction fibration, primary obstruction, Gysin sequence. 
any map $f: X \rightarrow X^{\prime}, \bar{f}: M\left(X^{\prime}, Y\right) \rightarrow M(X, Y)$ denotes the map defined by composition with $f$; i.e. $\bar{f}(\alpha)=\alpha \circ f$ for $\alpha \in M\left(X^{\prime}, Y\right)$.

Let $E^{2 m} \subset \mathbf{C}^{m}$ be the closed $2 m$-disc and $\iota: S^{2 m-1}=\dot{E}^{2 m} \hookrightarrow E^{2 m}$ the inclusion map. By restriction of maps to $\dot{E}^{2 m}$, we obtain a fibration

$$
\bar{\imath}: M\left(E^{2 m}, P^{n}\right) \rightarrow M\left(\dot{E}^{2 m}, P^{n}\right)
$$

with $\Omega^{2 m} P^{n}$ as fibre.

Similarly, the inclusion $\iota_{m-1}: P^{m-1} \hookrightarrow P^{m} \subset P^{n}, 1<m \leqslant n$, induces a fibration

$$
i_{m-1}^{k}: M_{k}\left(P^{m}, P^{n}\right) \rightarrow M_{k}\left(P^{m-1}, P^{n}\right) .
$$

This restriction fibration is the main object of study in this paper.

Since $P^{m}=P^{m-1} \cup E^{2 m}$ is the mapping cone of the canonical projection $p$ : $\dot{E}^{2 m}=S^{2 m-1} \rightarrow P^{m-1}$, the fibration $\bar{\iota}_{m-1}^{k}$ is the pull-back of $\bar{\imath}$ along the map $\bar{p}$ : $M_{k}\left(P^{m-1}, P^{n}\right) \rightarrow M\left(\dot{E}^{2 m}, P^{n}\right)$. It follows that $\bar{l}_{m-1}^{k}$ is an orientable fibration with fibre

$$
F_{m}^{k}=\left\{f \in M_{k}\left(P^{m}, P^{n}\right) \mid f \circ \iota_{m-1}=g_{k} \circ \iota_{m-1}\right\}
$$

homotopically equivalent to $\Omega^{2 m} P^{n}$. Here, and in the following, $g_{k}: P^{m} \rightarrow P^{m} \subset P^{n}$ is the cellular map of degree $k$ which in homogeneous coordinates is given by

$$
g_{k}\left[z_{0}: z_{1}: \cdots: z_{m}\right]=\left[z_{0}^{k}: z_{1}^{k}: \cdots: z_{m}^{k}\right] .
$$

For later use, we now calculate the effect of the map $\bar{g}_{k}: F_{m}^{1} \rightarrow F_{m}^{k}$ on the homotopy groups of the fibres.

LEMMA 2.1. The induced homomorphism $\left(\bar{g}_{k}\right)_{*}: \pi_{*}\left(F_{m}^{1}, g_{1}\right) \rightarrow \pi_{*}\left(F_{m}^{k}, g_{k}\right)$ is multiplication by $k^{m}$.

Proof. For any $f \in \Omega^{2 m} P^{n}$, let $g_{k}+f$ denote the composite map

$$
P^{m} \stackrel{\nu}{\rightarrow} P^{m} \vee S^{2 m} \stackrel{g_{k} \vee f}{\rightarrow} P^{n} \vee P^{n} \stackrel{\nabla}{\rightarrow} P^{n}
$$

where $\nabla$ is the folding map and $\nu$ is obtained by collapsing the boundary of an embedded $2 m$-disc $D^{2 m} \subset P^{m}$ to the base point. By choosing $D^{2 m}$ suitably, we get a homotopy commutative diagram

$$
\begin{array}{ccc}
\Omega^{2 m} P^{n} & \stackrel{\bar{f}_{k}}{\rightarrow} & \Omega^{2 m} P^{n} \\
g_{1}+\cdot \downarrow & & \downarrow g_{h}+\cdot \\
F_{m}^{1} & \stackrel{\bar{g}_{k}}{\rightarrow} & F_{m}^{k}
\end{array}
$$

in which the vertical maps are homotopy equivalences, cf. [5, p. 194], and $f_{k}$ : $S^{2 m}=P^{m} / P^{m-1} \rightarrow S^{2 m}=P^{m} / P^{m-1}$ is induced by $g_{k}$. Thus it suffices to show that $f_{k}$ has degree $k^{m}$. To that end, consider the commutative diagram of integral cohomology groups 


$$
\begin{array}{ccc}
H^{2 m}\left(P^{m} / P^{m-1}\right) & \stackrel{f_{k}^{*}}{\rightarrow} & H^{2 m}\left(P^{m} / P^{m-1}\right) \\
\cong \downarrow & \downarrow \cong \\
H^{2 m}\left(P^{m}, P^{m-1}\right) & \stackrel{g_{k}^{*}}{\rightarrow} & H^{2 m}\left(P^{m}, P^{m-1}\right) \\
\cong \downarrow & & \downarrow \cong \\
H^{2 m}\left(P^{m}\right) & \stackrel{g_{k}^{*}}{\rightarrow} & H^{2 m}\left(P^{m}\right)
\end{array}
$$

where the vertical homomorphisms, induced by natural inclusions and projections, are isomorphisms.

Let $c \in H^{2}\left(P^{m} ; \mathbf{Z}\right)$ be a generator. Then

$$
g_{k}^{*}\left(c^{m}\right)=\left(g_{k}^{*}(c)\right)^{m}=(k c)^{m}=k^{m} c^{m}
$$

since $g_{k}$ has degree $k$. As $c^{m}$ generates $H^{2 m}\left(P^{m}\right)$, this shows that $\operatorname{deg} f_{k}=k^{m}$.

Let $\mathrm{Ev}_{k}\left(P^{m}, P^{n}\right)$ denote the fibration

$$
e_{k}: M_{k}\left(P^{m}, P^{n}\right) \rightarrow P^{n}
$$

defined by evaluation at the base point $* \in P^{m}$; i.e. $e_{k}(f)=f(*)$ for $f \in$ $M_{k}\left(P^{m}, P^{n}\right)$. The fibre of $\mathrm{Ev}_{k}\left(P^{m}, P^{n}\right)$ is the space $F_{k}\left(P^{m}, P^{n}\right)$ of based maps of degree $k$ of $P^{m}$ into $P^{n}$. Also for these spaces of based maps, there are restriction fibrations

$$
i_{m-1}^{k}: F_{k}\left(P^{m}, P^{n}\right) \rightarrow F_{k}\left(P^{m-1}, P^{n}\right)
$$

with $\Omega^{2 m} P^{n}$ as fibre, $m>1$. Using this, an inductive argument yields

LeMMA 2.2. $F_{k}\left(P^{m}, P^{n}\right)$ is $(2 n-2 m)$-connected and $\pi_{2 n-2 m+1}\left(F_{k}\left(P^{m}, P^{n}\right)\right) \cong \mathbf{Z}$.

The Serre exact sequence for $\operatorname{Ev}_{k}\left(P^{m}, P^{n}\right)$ then shows

COROLlaRy 2.3. The induced homomorphism $e_{k}^{*}: H^{r}\left(P^{n}\right) \rightarrow H^{r}\left(M_{k}\left(P^{m}, P^{n}\right)\right)$ is an isomorphism for $0 \leqslant r \leqslant 2 n-2 m$.

3. Proof of Theorem. First assume $1<m<n$. Recall that

$$
i_{m-1}^{k}: M_{k}\left(P^{m}, P^{n}\right) \rightarrow M_{k}\left(P^{m-1}, P^{n}\right),
$$

as the pull-back of $\bar{\imath}: M\left(E^{2 m}, P^{n}\right) \rightarrow M\left(\dot{E}^{2 m}, P^{n}\right)$, is an orientable fibration with fibre $\Omega^{2 m} P^{n}$. By the Freudenthal Suspension Theorem, $\Omega^{2 m} P^{n}$ is equivalent to $S^{2 n-2 m+1}$ in dimensions $<4 n-4 m+1$. Hence $i_{m-1}^{k}$ has an associated Gysin sequence (integer coefficients) of the form

$$
\begin{aligned}
0 & \rightarrow H^{2 n-2 m+1}\left(M_{k}\left(P^{m}, P^{n}\right)\right) \rightarrow H^{0}\left(M_{k}\left(P^{m-1}, P^{n}\right)\right) \\
& \stackrel{\gamma^{k}}{\rightarrow} H^{2 n-2 m+2}\left(M_{k}\left(P^{m-1}, P^{n}\right)\right) \stackrel{\left(l_{m-1}^{k}\right)^{*}}{\rightarrow} H^{2 n-2 m+2}\left(M_{k}\left(P^{m}, P^{n}\right)\right) \rightarrow 0 .
\end{aligned}
$$

To get the trivial groups at the ends of this exact sequence we have used that $H^{2 n-2 m+1}\left(M_{k}\left(P^{m-1}, P^{n}\right)\right)=H^{1}\left(M_{k}\left(P^{m-1}, P^{n}\right)\right)=0$ by Corollary 2.3. In this Gysin sequence, $\gamma^{k}$ is cup product with the primary obstruction

$$
u^{k} \in H^{2 n-2 m+2}\left(M_{k}\left(P^{m-1}, P^{n}\right)\right) \cong \mathbf{Z}
$$


to constructing a cross-section of the fibration $\bar{l}_{m-1}^{k}$. The Theorem will follow once we have computed $u^{k}$. In fact, we only need to compute $u^{1}$, for the map $g_{k}$ : $\left(P^{m}, P^{m-1}\right) \rightarrow\left(P^{m}, P^{m-1}\right)$ of degree $k$ induces a fibre map

$$
\begin{array}{ccc}
M_{1}\left(P^{m}, P^{n}\right) & \stackrel{\bar{g}_{k}}{\rightarrow} & M_{k}\left(P^{m}, P^{n}\right) \\
\bar{i}_{m-1}^{1} \downarrow & & \downarrow \bar{i}_{m-1}^{k} \\
M_{1}\left(P^{m-1}, P^{n}\right) & \stackrel{\bar{g}_{k}}{\rightarrow} & M_{k}\left(P^{m-1}, P^{n}\right)
\end{array}
$$

of $\bar{l}_{m-1}^{1}$ into $\bar{l}_{m-1}^{k}$ and since

$$
\left(\bar{g}_{k}\right)^{*}: H^{2 n-2 m+2}\left(M_{k}\left(P^{m-1}, P^{n}\right)\right) \rightarrow H^{2 n-2 m+2}\left(M_{1}\left(P^{m-1}, P^{n}\right)\right)
$$

is an isomorphism by Corollary 2.3, we deduce from Lemma 2.1 that $u^{k}=k^{m} u^{1}$. We have, therefore, reduced the Theorem to the following

LEMMA 3.1. The formula $u^{1}= \pm\left(\begin{array}{c}n+1 \\ m\end{array}\right)$ holds for the primary obstruction $u^{1} \in$ $H^{2 n-2 m+2}\left(M_{1}\left(P^{m-1}, P^{n}\right)\right) \cong \mathbf{Z}$.

Before the proof of Lemma 3.1 we need a little preparation. With notation as in [5], let $\pi_{n+1, m}$ be the sphere bundle

$$
S^{2 n-2 m+1} \rightarrow U(n+1) / \Delta_{m+1} \times U(n-m) \rightarrow U(n+1) / \Delta_{m} \times U(n+1-m)
$$

over a quotient $W_{n+1, m} / \Delta_{m}=U(n+1) / \Delta_{m} \times U(n+1-m)$ of the complex Stiefel manifold $W_{n+1, m}=U(n+1) / U(n+1-m)$ by an action of the group $\Delta_{m} \cong$ $U(1)$. According to [5, Theorem 1.1], the primary obstruction $u^{1}$ agrees (up to sign) with the Euler class of $\pi_{n+1, m}$.

Let $\eta: W_{n+1, m} \rightarrow W_{n+1, m} / \Delta_{m}$ be the canonical principal $U(1)$-bundle and $\xi$ : $U(n+1) / \Delta_{m} \rightarrow W_{n+1, m} / \Delta_{m}$ the canonical principal $U(n+1-m)$-bundle over the base space $W_{n+1, m} / \Delta_{m}$ of $\pi_{n+1, m}$. With the aid of the complex vector bundles $\eta[\mathbf{C}]$ and $\xi\left[\mathbf{C}^{n+1-m}\right]$ associated to $\eta$ and $\xi$, respectively, we may give an alternative description of $\pi_{n+1, m}$.

LEMMA 3.2. The sphere bundle $\pi_{n+1, m}$ is fibre homotopically equivalent to the sphere bundle $S\left(\bar{\eta}[\mathbf{C}] \otimes \xi\left[\mathbf{C}^{n+1-m}\right]\right)$ of the tensor product of the conjugate bundle $\bar{\eta}[\mathbf{C}]$ of $\eta[\mathbf{C}]$ with $\xi\left[\mathbf{C}^{n+1-m}\right]$.

Taking this for granted we return to the

Proof OF LemMa 3.1. Let $c(\eta)=1+c_{1}(\eta)$ and $c(\xi)=1+\sum c_{i}(\xi)$ be the total Chern classes of $\eta$ and $\xi$. As $W_{n+1, m}$ is $(2 n-2 m+2)$-connected, the infinite cyclic group $H^{2 i}\left(W_{n+1, m} / \Delta_{m} ; \mathbf{Z}\right)$ is generated by $c_{1}(\eta)^{i}$ for $i \leqslant n+1-m$. Thus $c(\xi)$ can be expressed by the powers of $c_{1}(\eta)$. In fact,

$$
c_{i}(\xi)=\left(\begin{array}{c}
-m \\
i
\end{array}\right) c_{1}(\eta)^{i}, \quad 1 \leqslant i \leqslant n+1-m,
$$

for it is easily seen that the Whitney sum $\eta[\mathbf{C}]^{m} \oplus \xi\left[\mathbf{C}^{n+1-m}\right]$ is trivial. 
Since $u^{1}$ is the Euler class of $\pi_{n+1, m}$ we conclude, using Lemma 3.2, that up to sign

$$
\begin{aligned}
u^{1} & =c_{n+1-m}\left(\bar{\eta}[\mathbf{C}] \otimes \xi\left[\mathbf{C}^{n+1-m}\right]\right)=\sum_{i=0}^{n+1-m} c_{1}(\bar{\eta})^{n+1-m-i} \cup c_{i}(\xi) \\
& =\sum_{i=0}^{n+1-m}(-1)^{i}\left(\begin{array}{c}
-m \\
i
\end{array}\right) c_{1}(\eta)^{n+1-m}=\left(\begin{array}{c}
n+1 \\
m
\end{array}\right) c_{1}(\eta)^{n+1-m} .
\end{aligned}
$$

Proof of Lemma 3.2. Let $\Delta_{m} \times U(n+1-m)$ act on $\mathbf{C} \otimes \mathbf{C}^{n+1-m}=\mathbf{C}^{n+1-m}$ by $(z, B) \cdot v=\bar{z} B v$ for $z \in \Delta_{m}=S^{1} \subset \mathbf{C}, B \in U(n+1-m)$, and $v \in \mathbf{C}^{n+1-m}$. Then the stabilizer of the basis vector $e_{m+1} \in\{0\} \times \mathbf{C}^{n+1-m} \subset \mathbf{C}^{n+1}$ is $\Delta_{m+1} \times$ $U(n-m)$, so since the diagonal map

$$
D: U(n+1) \rightarrow U(n+1) / U(n+1-m) \times U(n+1) / \Delta_{m}
$$

is a $\Delta_{m} \times U(n+1-m)$-map, it follows that

$$
D \times e_{m+1}: U(n+1) \rightarrow U(n+1) / U(n+1-m) \times U(n+1) / \Delta_{m} \times S^{2 n-2 m+1}
$$

induces a fibre homotopy equivalence

$$
\pi_{n+1-m} \cong d^{*}(\eta \times \xi)\left[S^{2 n-2 m+1}\right]
$$

between $\pi_{n+1, m}$ and the pull-back along the diagonal map $d$ of $W_{n+1, m} / \Delta_{m}$ of the fibre bundle $(\xi \times \eta)\left[S^{2 n-2 m+1}\right]$ associated to the principal $\Delta_{m} \times U(n+1-m)$ bundle $\xi \times \eta$ over $W_{n+1, m} / \Delta_{m} \times W_{n+1, m} / \Delta_{m}$. But clearly,

$$
d^{*}(\eta \times \xi)\left[S^{2 n-2 m+1}\right]=S\left(d^{*}(\eta \times \xi)\left[\mathbf{C}^{n+1-m}\right]\right)=S\left(\bar{\eta}[\mathbf{C}] \otimes \xi\left[\mathbf{C}^{n+1-m}\right]\right) .
$$

The Theorem has now been proved for $1<m<n$. The case $1<m \leqslant n$ is covered by $[2,4]$, but may also be obtained by some minor changes in the above proof. For $m=n$, a slightly stronger statement holds.

Proposition 3.3. The fundamental group of $M_{k}\left(P^{n}, P^{n}\right), n \geqslant 1$, is cyclic of infinite order for $k=0$ and cyclic of order $(n+1)|k|^{n}$ for $k \neq 0$ :

$$
\pi_{1}\left(M_{k}\left(P^{n}, P^{n}\right)\right) \cong \mathbf{Z} /(n+1)|k|^{n} \mathbf{Z} \text {. }
$$

Proof. As the case $n=1$ was handled in [4] we may assume that $n>1$. Composition with $g_{k}:\left(P^{n}, P^{n-1}\right) \rightarrow\left(P^{n}, P^{n-1}\right)$ defines a fibre map $\bar{g}_{k}$ of $i_{n-1}^{1}$ into $\bar{i}_{n-1}^{k}$ which induces a map

$$
\begin{aligned}
& \rightarrow \pi_{2}\left(M_{1}\left(P^{n-1}, P^{n}\right)\right) \stackrel{\partial_{1}}{\rightarrow} \pi_{1}\left(F_{n}^{1}\right) \quad \rightarrow \quad \pi_{1}\left(M_{1}\left(P^{n}, P^{n}\right)\right) \quad \rightarrow \quad 0 \\
& m_{2} \downarrow \quad \downarrow m_{1} \\
& \rightarrow \pi_{2}\left(M_{k}\left(P^{n-1}, P^{n}\right)\right) \stackrel{\partial_{2}}{\rightarrow} \pi_{1}\left(F_{n}^{k}\right) \quad \rightarrow \quad \pi_{1}\left(M_{k}\left(P^{n}, P^{n}\right)\right) \quad \rightarrow \quad 0
\end{aligned}
$$

between the homotopy sequences. Since

$$
\left(e_{r}\right)_{*}: \pi_{2}\left(M_{k}\left(P^{n-1}, P^{n}\right)\right) \rightarrow \pi_{2}\left(P^{n}\right), \quad r=1, k,
$$

is an isomorphism by Lemma 2.2, it follows that $m_{2}$ is an isomorphism. As $\pi_{1}\left(M_{1}\left(P^{n}, P^{n}\right)\right)=\mathbf{Z}_{n+1}$ by [4], $\partial_{1}$ must be multiplication by $n+1$. Using Lemma 2.1, we see that $\partial_{2}=\partial_{2} \circ m_{2}=m_{1} \circ \partial_{1}$ is multiplication by $(n+1) k^{n}$. Hence

$$
\pi_{1}\left(M_{k}\left(P^{n}, P^{n}\right)\right) \cong \pi_{1}\left(F_{n}^{k}\right) / \mathrm{im} \partial_{2} \cong \mathbf{Z} /(n+1)|k|^{n} \mathbf{Z} .
$$




\section{REFERENCES}

1. V. L. Hansen, The homotopy problem for the components in the space of maps on the n-sphere, Quart. J. Math. Oxford Ser. (3) 25 (1974), 313-321.

2. __ On the space of maps of a closed surface into the 2-sphere, Math. Scand. 35 (1974), 149-158.

3. 273-281.

4. S. T. Hu, Concerning the homotopy groups of the components of the mapping space $Y^{S}$, Indag. Math. 8 (1946), 623-629.

5. S. Sasao, The homotopy of $\operatorname{Map}\left(C P^{m}, C P^{n}\right)$, J. London Math. Soc. (2) 8 (1974), 193-197.

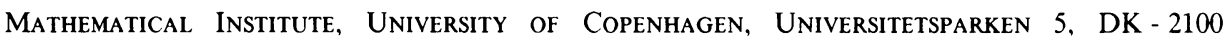
COPENHAGEN $\phi$, DENMARK 